chromosome movements (the question was not settled). The possibilities of artefacts in preparing cells for antibody or heavy meromyosin (HMM) labelling was keenly discussed and although there is growing evidence for the presence of actin, myosin has not been demonstrated. The studies of $\mathrm{L}$. G. Tilney (University of Pennsylvania) have shown that actin is present on its own in the acrosome bodies of sperm from a variety of species and is driven into the egg by polymerisation or uncoiling. Thus the presence of actin in a cell organelle need not imply the presence of myosin as well.

Some three years ago, R. C. Weisenberg (Temple University, Philadelphia) developed a method of polymerisation of tubulin into microtubules. This work stimulated an intense study of the mechanism of microtubule polymerisation and the search for microtubule organising centres. Condensation of tubulin into outer doublets of flagella and asters of the mitotic apparatus has already been described and evidence was presented (by two different laboratories) for the chromosome kinetochore as an organiser. Microtubule polymerisation has turned out to be a complex process, more like virus assembly than the type of spontaneus polymerisation that occurs with fibrous proteins (actin for example). The formation of a ring structure by a protein factor or possibly polyvalent ions, the use of the ring as a nucleating centre, the participation of high molecular weight factors, and enzymes (transphosphorylase and possibly a protein kinase), the requirement for nucleotide triphosphate hydrolysis, as well as three different polymerisation models $(H$. $P$. Erickson-M. Kirschner (Duke University). G. G. Borisy (University of Wisconsin), M. Jacobs et al. (King's College, London)) were discussed. While it is clear that rapid progress is being made on this problem, more rigorous purification of the system is necessary before the discrepancies between different groups can be resolved.

\section{Transient observations of X-ray sources}

from John Gribbin

At the Royal Astronomical Society meeting on October 10 the nature of the transient X-ray source Ariel 0620-00 was discussed.

THE discovery of the source early in August was described by $M$. Turner (University of Leicester). There was a rapid rise in intensity, with one slight hiccup, which made A0620-00 three times brighter than Sco X-1 by the middle of the month (see Nature, 257, $656,657 ; 1975)$. The hiccup, or 'precursor peak' seems to have marked a change in the energy production mechanism, with a fall in temperature preceeding the most rapid period of increasing brightness, and with the bulk of the increase occurring at low energy (below $10 \mathrm{keV}$ ) making this a very soft source. No lines have been found in the spectrum, although searches have been made especially at frequencies appropriate for ionised silicon and sulphur, and there is no evidence of polarisation at what Turner described as "the lowest upper limit yet achieved in X-ray astronomy" and there is no evidence of periodic variations anywhere in the range $0.2 \mathrm{~ms}$ to $2 \mathrm{~d}$.

But there are some positive interpretations of the X-ray evidence, not least a SAS 3 measurement of the hydrogen column density to the source which implies a distance of $3 \mathrm{kpc}$ if all absorption is interstellar, suggesting an absolute luminosity of $10^{39} \mathrm{erg} \mathrm{s}^{-1}$.

A0620-00 has also been detected at radiofrequencies and identified with an optical star (see Nature, 257, 659; 1975). R. Davis (Jodrell Bank) described radio observations both at Manchester and elsewhere which help to provide a very accurate position in right ascension, although the declination is less well determined because of the inconvenient position of the object for such measurements. The source is variable, and declined from peak intensity after August 18 with an $e$-folding time of $5 \mathrm{~d}$, considerably faster than the equivalent X-ray decline (1/e fall off over $30 \mathrm{~d}$ ). Assuming the outburst began on August 3, the radio data can be fitted by a $T^{-3}$ power low.

The optical observations were described by $M$. Penston (Royal Greenwich Observatory), who preferred to call the event Nova Monoceros 1975 but agreed that he was indeed talking about the same thing. There seems no doubt at all about the identification of the optical and X-ray novae, and comparison with Palomar Sky Survey plates shows that during the outburst the source increased from a magnitude of 20.5 to 11.5 , a brightening which is on the high side for dwarf novae but not unprecedented. The Palomar plates also show that the star was very red before the outburst, suggesting that it may be a red dwarf only some 500 pc away or a giant at the implausible distance of $15 \mathrm{kpc}$ which, in the direction of Monoceros, would place it not only well out of the Galaxy but far above the galactic plane. At optical frequencies, the fade of the source has been little slower than in the X-ray band but faster than at radio frequencies, indicated by measurements up to a few days before the RAS meeting.

For the modellers, one of the most significant contributions comes from a search of Harvard plates which show a previous outburst in 1971, reaching $12 \mathrm{mag}$. This strongly implies the presence of circumstellar material leftover from that event, suggesting that the SAS 3 distance must be an upper limit and pinning the object down clearly as a red dwarf probably closer than $1 \mathrm{kpc}$.

A. Fabian (Institute of Astronomy, Cambridge) took up this theme in presenting a model of A0620-00. Pointing out that this is the first optical identification of a transient X-ray source, he made a leap which perplexed some optical astronomers-but not the X-ray specialists-by assuming the now basic model of a binary source with mass transfer from the visible star onto a compact companion, probably a neutron star. The probability of seeing an eclipse on such a model is only $12 \%$, so it is not surprising that none is seen in this source, and the increase in brightness of the dwarf is caused by the heating effect of X-radiation from the companion on one face of the larger star. The fact that novae seem to recur in this system encouraged Fabian to make a comparison with the recurrent nova WZ Sge, itself a similar binary system, and although this detailed model is unlikely to fit all the transient $\mathrm{X}$-ray sources it is interesting that a very similar model has already been proposed for the archetypal X-ray source Sco X-1, again drawing a comparison with WZ Sge.

This raises the question of just how different the transient X-ray sources are from the rest of the family. All of the known X-ray sources that are not supernova remnants show very erratic behaviour with flares and quieter periods, and if Sco X-1 can be explained, after more than 10 years observation, by a model similar to that appropriate for a newly discovered source which flared into prominence for only a few weeks it seems that the differences may well be of degree rather than of kind. The observations themselves are far from continuous, and the label hung upon any particular source can depend to a large extent on the state it happened to be in when it was first noticed. Perhaps the main lesson to be learned from the story of A0620-00 so far is that all X-ray binaries are variable, but some vary more than others, and that rather than talking about "observations of transient X-ray sources" we would do better to talk about "transient observations of X-ray sources". 